\title{
Stochastic simulations for the time evolution of systems which obey generalized statistics: Fractional exclusion statistics and Gentile's statistics
}

\author{
G. A. Nemnes \\ University of Bucharest, Faculty of Physics, "Materials and Devices for Electronics \\ and Optoelectronics" Research Center, P.O. Box MG-11, 077125 Magurele-Ilfov, \\ Romania \\ E-mail: nemnes@solid.fizica.unibuc.ro \\ D. V. Anghel \\ "Horia Hulubei" National Institute for Physics and Nuclear Engineering (IFIN-HH), \\ 077126 Magurele-Ilfov, Romania \\ E-mail: dragos@theory.nipne.ro
}

\begin{abstract}
We present a stochastic method for the simulation of the time evolution in systems which obey generalized statistics, namely fractional exclusion statistics and Gentile's statistics. The transition rates are derived in the framework of canonical ensembles. This approach introduces a tool for describing interacting fermionic and bosonic systems in non-equilibrium as ideal FES systems, in a computationally efficient manner. The two types of statistics are analyzed comparatively, indicating their intrinsic thermodynamic differences and revealing key aspects related to the species size.
\end{abstract}




\section{Introduction}

Two of the best known generalizations of the Bose and Fermi statistics are the Gentile's statistics (GS) [1, 2, 3] and the fractional exclusion statistics (FES) [4]. A method for the stochastic simulation of the time evolution of Bose and Fermi gases was proposed by Guastella et al. in Ref. [5]. In this paper we present a method for the simulation of the more general GS and FES systems, which have as limit cases the Fermi and Bose statistics. As we shall see below, the GS and the FES are apparently closely related. Nevertheless they give totally different thermodynamic results.

The GS describes systems of non-interacting particles in which each single particle state can be occupied by a maximum of $\xi$ particles; the case $\xi=1$ corresponds to the Fermi statistics and the case $\xi=\infty$ corresponds to the Bose statistics.

The definition of FES is somewhat more complicated. A FES system is formed in general of several subsystems, called species. Each species consists of a finite number of particles in a finite dimensional space, spanned by a set of single-particle quantum numbers. They are defined by coarse-graining the set of single-particle quantum numbers for each type of particles in the system. Each species has a certain number of available single-particle states which may depend, in principle, on the number of particles in any of the species of the system. This dependence is known as the "statistical interaction" between the FES particles and limits the number of particles that can be accommodated in each species, similarly as in the GS.

Let us number the species by $i=0,1, \ldots$ and denote by $N_{i}$ and $G_{i}$ the number of particles and the number of available single-particle states of species $i$, respectively. The statistical interaction is expressed by the change in the number of available singleparticle states at the change of the particle numbers. For example a change $\delta N_{i}$ of $N_{i}$ will change $G_{j}$ by $-\alpha_{j i} \delta N_{i}$, for any $j$. The FES parameters, $\alpha_{i j}$, depend on the way the system is divided into species - different divisions lead to different $\alpha$ 's [6]. For non-interacting bosons and fermions, $\alpha_{i j}=0$ and $\alpha_{i j}=\delta_{i j}$, respectively, for any $i$ and $j$.

Now the connection between GS and FES is obvious. If we would have two similar types of systems, one described by GS, of parameter $\xi$, and the other one described by FES, of parameters $\alpha_{i j}=\delta_{i j} \alpha \equiv \delta_{i j} / \xi$, we may coarse-grain the sets of quantum numbers that describe these systems and obtain the "species" $i=0,1, \ldots$, each with $N_{i}$ particles and $G_{i}$ available states. If we denote by $G_{i}^{(0)}$ the number of available states when $N_{i}=0$, for any $i$, then the maximum number of particles that we can put into the species $i$ in both gases is the same, namely $\xi G_{i}^{(0)}=G_{i}^{(0)} / \alpha$. Based on this single observation we could say that GS is pure FES with species formed of single-particle states.

Nevertheless, the thermodynamics of FES and GS systems are different. In general, FES is applied to species of large numbers of particles and states and cannot be applied to single states, like GS. If one would attempt to make the FES species smaller and smaller, not only that the thermodynamic results deviate from those obtained with 
large species, as we shall show below, but the very definition of FES becomes unclear. On the other hand, if we apply both, FES and GS to particle species-say we have the species $\left\{N_{i}, G_{i}\right\}_{i=0,1, \ldots}$-the number of microconfigurations in which the particles can arrange themselves is not the same in FES [4, 7, 8, 9, 10] and GS [1, 2, 3] systems. Therefore the thermodynamic results must be different.

The difference between the FES and the GS is very clearly evidenced in systems of constant density of states (DOS). It is well known that Bose, Fermi and, in general, FES systems of constant DOS are thermodynamically equivalent, i.e. the specific heat and entropy are independent of the statistics [11, 12, 13, 14, 15, 16]. The microscopic reason of this equivalence lies in the fact that one can realize a one-to-one mapping between microconfigurations of particles of the same excitation energies across all statistics by the "excitation energy" of a microconfiguration we mean the difference between the energy of the microconfiguration and the lowest energy of the system [15]. As we shall see in the following, the GS does not fall into this equivalence class. For a GS of constant DOS, the specific heat, and therefore also the entropy, depend on the parameter $\xi$.

As stated above, we propose in this paper a method to stochastically simulate the time evolution of GS and FES systems. The paper is organized as follows. For the clarity of the exposition, we start in Section 2 by briefly reviewing the method of Guastella et al.. Then we extend the method to the FES and GS gases in Section 3. Finally, in Section 4 the derived transition probabilities associated with the Markov chain that we introduce are used in simulations of FES and GS systems, the results are tested against corresponding analytical data and particularities of the two statistics are commented upon.

\section{Simulation of Bose and Fermi ideal gases}

Let us consider a system of ideal bosons or fermions in contact with a heat bath, at temperature $T$. The single particle states of the system, denoted by $|i\rangle$, have the energies $\epsilon_{i}(i=0,1, \ldots)$; the energy levels may be degenerated.

The dynamics of the system is described in terms of a time dependent Markov chain in the microconfiguration space. Each microconfiguration is a set $\left\{n_{i}\right\}_{i=0,1, \ldots}$, where $n_{i}$ is the particle population of the state $|i\rangle$. For fluency we shall call the microconfigurations simply states, which must not be confused with the single-particle states. The system evolves in time from one state to another neighboring state by random jumps of particles between the single-particle states. The transition rates between two states will be denoted by $T_{\left\{n_{i}^{\prime}\right\},\left\{n_{i}\right\}}$, where the notation convention is that the first subscript refers to the final state and the second subscript refers to the initial state. Assuming that in the dynamics of the system the particles jump between single particle states one at a time, we shall consider that two states are neighbors if they differ by the exchange of only one particle between two single particle states. Therefore, we shall use the simpler notation $T_{j i}\left(n_{j}, n_{i}\right)$ to refer to the transition rate of a particle from the state $|i\rangle$, of $n_{i}$ particles, to the state $|j\rangle$, of $n_{j}$ particles. 
If the particles are bosons, the Fermi golden rule imposes in general that $T_{j i}\left(n_{j}, n_{i}\right)$ is proportional to $\left(n_{j}+1\right) n_{i}$. For fermions, the Pauli exclusion principle gives a transition rate $T_{j i} \propto\left(1-n_{j}\right) n_{i} \equiv n_{i} \delta_{n_{j}, 0}$, since each state can accommodate no more than one particle. As the system is in contact with a thermostat, the relative transition rate, $T_{j i}\left(n_{j}, n_{i}\right) / T_{i j}\left(n_{i}, n_{j}\right)$, must also be proportional to a weight factor, $e^{-\beta\left(\epsilon_{j}-\epsilon_{i}\right)}$, where $\beta \equiv 1 /\left(k_{B} T\right)$.

After setting-up the initial microconfiguration, the simulation proceeds by allowing the particles to make random jumps between the single-particle states, with probabilities proportional to the transition rates. In what follows we shall denote by $p_{\left\{n_{i}\right\}}$ the probability to find the system in the microconfiguration $\left\{n_{i}\right\}$. Then the mean occupation of a single particle state is

$$
\left\langle n_{j}\right\rangle=\sum_{\left\{n_{i}\right\}} p_{\left\{n_{i}\right\}} n_{j} .
$$

After the system is simulated long enough, the equilibrium is reached and the ensemble averages of the thermodynamic quantities do not change in time anymore. Once this condition is reached, the equilibrium probabilities, $p_{\left\{n_{i}\right\}}^{\mathrm{Eq}}$, satisfy the detailed balance equation (DBE),

$$
p_{\left\{n_{k}\right\}}^{\mathrm{Eq}} T_{\left\{n_{k}^{\prime}\right\},\left\{n_{k}\right\}}=p_{\left\{n_{k}^{\prime}\right\}}^{\mathrm{Eq}} T_{\left\{n_{k}\right\},\left\{n_{k}^{\prime}\right\}} .
$$

for any neighboring states, $\left\{n_{k}\right\} \equiv\left(\ldots, n_{i}, \ldots, n_{j}, \ldots\right)$ and $\left\{n_{k}^{\prime}\right\} \equiv\left(\ldots, n_{i}^{\prime}, \ldots, n_{j}^{\prime}, \ldots\right) \equiv$ $\left(\ldots, n_{i}-1, \ldots, n_{j}+1, \ldots\right)$. On the other hand, since the system is in contact with a heat bath, the equilibrium probabilities should be the canonical probabilities,

$$
p_{\left\{n_{i}\right\}}^{\mathrm{Eq}}=e^{-\beta\left[\sum_{i} n_{i} \epsilon_{i}\right]} / Z(T, N),
$$

where $Z(T, N)$ is the canonical partition function.

The DBE can be easily checked. If we plug the expression (3) into (2), with $T_{\left\{n_{k}^{\prime}\right\},\left\{n_{k}\right\}} \equiv T_{j i}\left(n_{j}, n_{i}\right) \propto\left(1 \pm n_{j}\right) n_{i}$, we obtain an identity. Therefore the procedure described above produces the canonical probabilities when equilibrium is reached.

\section{Simulation of systems which obey intermediate statistics}

\subsection{Fractional exclusion statistics}

The calculation of the transition rates in FES systems is not as straightforward as in the Bose and Fermi gases. A FES system is essentially an interacting particle system, described by a (countable) set of "quasiparticle" quantum numbers, $\mathbf{k}_{i}$, where $i=0,1, \ldots$ [4, 17, 8, 17, 18, 10]. For example 1D integrable quantum gases of interacting bosons and fermions [19, 20, 10] and systems described by the Fermi liquid model [21, 22, 15, 23, 17, 10] are typical FES systems. For the 1D integrable quantum gases the quantum numbers are the asymptotic momenta, $k_{i}$, whereas for the Fermi liquid systems the "quasiparticle" energies, $\tilde{\epsilon}_{i}$, are used as quantum numbers. The population

of the "quasiparticle" states will be denoted by $n_{i}$ or $n\left(k_{i}\right)$. The particles are interacting 
and therefore the values of the quantum numbers may depend (in principle) on the whole set $\left\{n_{i}\right\}_{i=0,1, \ldots}$, which defines also the quantum state of the system.

To show how the dependence of $\mathbf{k}_{i}$ on $\left\{n_{j}\right\}_{j=0,1, \ldots}$ leads to FES (see also [18] for more details), let us assume that the allowed values of $\mathbf{k}_{i},\left\{\mathbf{k}_{i}\right\}_{i=0,1, \ldots}$, form a set of points in a $d$-dimensional $(d \mathrm{D})$ vector space. To build a statistical description of the system, we have to calculate the number of configurations in which the particles can occupy different one-particle states at constant total energy $E$ and particle number $N$. For this, we divide the $d \mathrm{D}$ space of $\mathbf{k}$ vectors into elementary, fixed volumes, $V_{i}$, each containing $G_{i}$ states and $N_{i}$ particles - these are our species and should be made no confusion from using the same subscripts, $i, j$, etc. for counting both, the states, $\left|\mathbf{k}_{i}\right\rangle$, and the species, $V_{i}, G_{i}, N_{i}$. If the particles in the system are bosons, $n_{i}$ may take any positive integer value and the number of microconfigurations we have in the system for this division into species is

$$
W_{B}\left(\left\{G_{i}, N_{i}\right\}\right)=\prod_{i} \frac{\left(G_{i}+N_{i}-1\right) !}{N_{i} !\left(G_{i}-1\right) !} .
$$

If the particles are fermions, $n_{i}=0$ or 1 and the total number of microconfigurations is

$$
W_{F}\left(\left\{G_{i}, N_{i}\right\}\right)=\prod_{i} \frac{G_{i} !}{N_{i} !\left(G_{i}-N_{i}\right) !} .
$$

Since in our system the values $\left\{\mathbf{k}_{i}\right\}$ change with the particle populations, $\left\{n_{i}\right\}$, while the elementary volumes, $V_{i}$, are fixed in the $\mathbf{k}$ space, this leads to a dependence of the $G_{i}$ 's on the set $\left\{N_{j}\right\}_{j=0,1, \ldots}[9,17,18,10]$.

To quantify this statement, let us assume that a small change, $\delta N_{i}$, of $N_{i}$ produces a linear change,

$$
\delta G_{j}=-\alpha_{j i} \delta N_{i},
$$

of the number of states, $G_{j}$, for any $i$ and $j$ (not all the $\alpha$ 's have to be different from zero). The proportionality constants, $\alpha_{i j}$, are the FES parameters defined in the Introduction.

Using Eqs. (3.1) and (5) we can calculate the grandcanonical partition function,

$$
\mathcal{Z}_{p}^{\mathrm{FES}}\left(\left\{G_{i}, N_{i}\right\}\right)=\sum_{\left\{N_{i}\right\}} e^{\beta \sum_{i} N_{i}\left(\mu-\epsilon_{i}\right)} W_{p}\left(\left\{G_{i}, N_{i}\right\}\right)
$$

where we assumed that the total energy of the system is $E\left(\left\{N_{i}\right\}\right) \equiv \sum_{i} N_{i} \epsilon_{i}$. This is equivalent to ascribing the same energy-the average energy-to all the particles in a species. In Eq. (6) $p$ stands for $B$ or $F$, depending on whether we have bosons or fermions in the system. Using Eq. (6) we can calculate the equilibrium properties of the system by maximizing $\mathcal{Z}^{\text {FES }}$ with respect to the set $\left\{N_{i}\right\}$ [7, 10]. In this way we obtain for bosons and fermions the systems of equations

$$
\begin{aligned}
& \frac{1+\tilde{n}_{i}}{\tilde{n}_{i}} \prod_{j}\left(1+\tilde{n}_{j}\right)^{-\alpha_{j i}}=e^{\beta\left(\epsilon_{i}-\mu\right)} \\
& \frac{1-\tilde{n}_{i}}{\tilde{n}_{i}} \prod_{j}\left(1-\tilde{n}_{j}\right)^{\alpha_{j i}}=e^{\beta\left(\epsilon_{i}-\mu\right)},
\end{aligned}
$$


respectively, where $\tilde{n}_{i} \equiv N_{i} / G_{i}$. One should note that in both Eqs. (17a) and (7b) $\alpha_{j i}=0$ corresponds to non-interacting bosonic and fermionic systems, respectively.

To calculate the transition rates, we have to consider separately the systems of bosons and the systems of fermions; we shall be interested in transitions in which a particle jumps from one species to another, therefore changing the particle distribution over the species.

Let's consider the jump of one particle from the species $i$ into the species $j$ and vice-versa, in a system of bosons. For the jump $i \rightarrow j$, we start from a configuration of $N_{i}$ particles and $G_{i}$ available states in species $i$ and arrive into a configuration of $N_{j}$ particles and $G_{j}$ states in the species $j$. The reversed process, $j \rightarrow i$, is exactly like $i \rightarrow j$, but in reverse order, i.e. we start from a configuration of $N_{j}$ particles and $G_{j}$ states in the species $j$ and arrive in a configuration of $N_{i}$ particles and $G_{i}$ states in the species $i$.

Let's now focus on the process $i \rightarrow j$ and assume that the $N_{i}$ particles are distributed as $n_{i_{0}}, n_{i_{1}}, \ldots$ onto the states with the quantum numbers $\mathbf{k}_{i_{0}}, \mathbf{k}_{i_{1}}, \ldots$ Analogously, the $N_{j}-1$ particles that exist in the species $j$ before the jump, are distributed as $n_{j_{0}}, n_{j_{1}}, \ldots$ particles on the states $\mathbf{k}_{j_{0}}, \mathbf{k}_{j_{1}}, \ldots$ Keeping the notations from Section 2, we have the transition rates $T_{j_{m} i_{n}}\left(n_{j_{m}}, n_{i_{n}}\right) \propto\left(n_{j_{m}}+1\right) n_{i_{n}}$. Therefore the transition rate of a particle-any particle-from the species $i$ to the state $\mathbf{k}_{j_{m}}$ of species $j$ is $T_{j_{m} i}\left(n_{j_{m}}, N_{i}\right) \propto\left(n_{j_{m}}+1\right) N_{i}$. Moreover, the transition of a particle from the species $i$ onto any state of the species $j$ can be found by summing-up over all the $G_{j}$ states of the species $j$, leading to $T_{B, j i}\left(N_{j}, N_{i}\right) \propto\left(G_{j}+N_{j}-1\right) N_{i}$.

Further, to obtain the total transition rate from the species $i$ to the species $j$, we have to multiply the above result with the number of microconfigurations, $W_{B, i j}=\left(G_{j}+N_{j}-2\right) ! /\left[\left(N_{j}-1\right) !\left(G_{j}-1\right) !\right] \times\left(G_{i}+N_{i}-1\right) ! /\left[N_{i} !\left(G_{i}-1\right) !\right]$. In this way we finally obtain, up to a common multiplicative factor and the canonical weight, $e^{\beta\left(\epsilon_{i}-\epsilon_{j}\right)}$,

$$
T_{B, j i}\left(N_{j}-1, N_{i}\right)=\frac{\left(G_{j}+N_{j}-1\right) !}{\left(N_{j}-1\right) !\left(G_{j}-1\right) !} \frac{\left(G_{i}+N_{i}-1\right) !}{\left(N_{i}-1\right) !\left(G_{i}-1\right) !} .
$$

The DBE then reads,

$e^{-\beta\left[N_{i} \epsilon_{i}+\left(N_{j}-1\right) \epsilon_{j}\right]} T_{B, j i}\left(N_{j}-1, N_{i}\right) e^{\beta\left(\epsilon_{i}-\epsilon_{j}\right)}=e^{-\beta\left[\left(N_{i}-1\right) \epsilon_{i}+N_{j} \epsilon_{j}\right]} T_{B, i j}\left(N_{i}-1, N_{j}\right)$

which, by using Eq. (8), it can be immediately checked to be an identity.

A similar argument can be used for transitions in systems of fermions, with the difference that the particles cannot arrive on a quantum state already occupied. This leads to a transition rate $T_{F, j i}\left(N_{j}, N_{i}\right) \propto\left(G_{j}-N_{j}\right) N_{i}$. Multiplying again by the number of microconfigurations, we obtain

$$
T_{F, j i}\left(N_{j}-1, N_{i}\right)=\frac{G_{j} !}{\left(N_{j}-1\right) !\left(G_{j}-N_{j}\right) !} \frac{G_{i} !}{\left(N_{i}-1\right) !\left(G_{i}-N_{i}\right) !} .
$$

Like in the Bose systems, the transition rates (10) satisfy identically the DBE. 


\subsection{Gentile's statistics}

In GS each single-particle state can be occupied by up to $\xi$ particles. This can be described in a second quantized theory by introducing the creation and annihilation operators $a_{\mathbf{k}}^{\dagger}$ and $a_{\mathbf{k}}$, respectively, of the form [2]

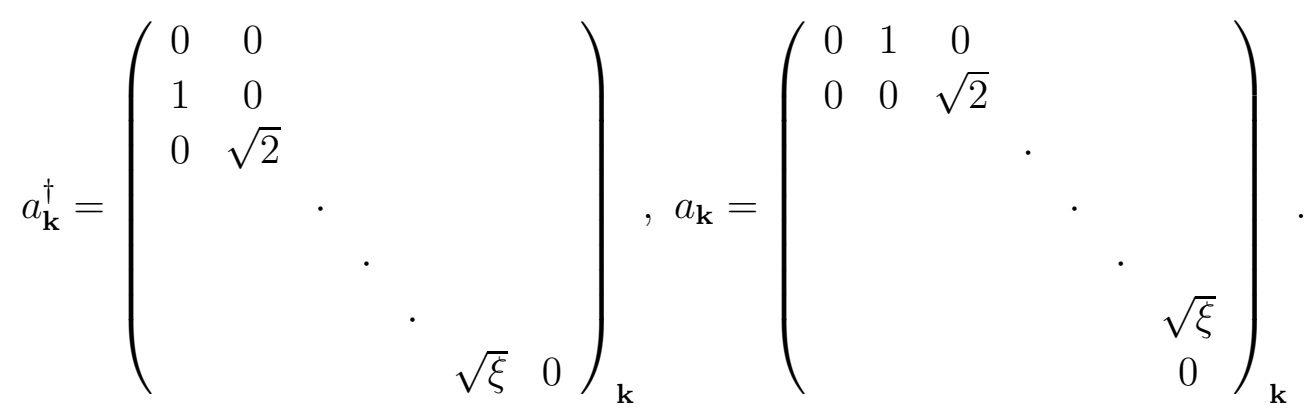

The creation and annihilation operators are assumed to commute for different $\mathbf{k}$ 's. If we denote by $|0\rangle$ the vacuum state, then a many-body state of $N=n_{\mathbf{k}_{0}}+n_{\mathbf{k}_{1}}+\ldots$ particles is $\left|n_{\mathbf{k}_{0}}, n_{\mathbf{k}_{1}}, \ldots\right\rangle=\left(a_{\mathbf{k}_{0}}^{\dagger}\right)^{n_{\mathbf{k}_{0}}}\left(a_{\mathbf{k}_{1}}^{\dagger}\right)^{n_{\mathbf{k}_{1}}} \ldots|0\rangle$.

As the system is connected to a heat bath, the eigenstates of the free GS particle system are perturbed by external interactions. The transition rate of a particle from the state $\left|\mathbf{k}_{i}\right\rangle$ to the state $\left|\mathbf{k}_{j}\right\rangle, T_{j i}$, caused by this interaction should be proportional

to $a_{\mathbf{k}_{j}}^{\dagger} a_{\mathbf{k}_{i}}$. Using (11), we obtain $T_{j i} \propto\left(n_{\mathbf{k}_{j}}+1\right) n_{\mathbf{k}_{i}}$, like in the case of bosons, with the extra condition that $T_{j i}=0$ if $n_{\mathbf{k}_{j}} \geq \xi$. These transition rates obey the DBE, like in Section 2 ,

The thermal properties of the GS have been calculated in several papers (see for example Ref. [2]). We assume that the single-particle states $\left|\mathbf{k}_{i}\right\rangle$ are eigenstates of the Hamiltonian of the (ideal) system, with the eigenvalues $\epsilon_{i}$ (or, equivalently, $\epsilon_{\mathbf{k}_{i}}$ ). Since only up to $\xi$ particles can occupy at same time such a state, the partition function can be written as

$$
\mathcal{Z}^{(G S)}=\prod_{i}\left[\sum_{j=0}^{\xi} e^{j \beta\left(\mu-\epsilon_{i}\right)}\right] \equiv \prod_{i}\left[\frac{1-e^{(\xi+1) i \beta\left(\mu-\epsilon_{i}\right)}}{1-e^{\beta\left(\mu-\epsilon_{i}\right)}}\right]
$$

From Eq. (12a $)$ we can calculate the grandcanonical thermodynamic potential,

$$
\Omega^{(G S)}=-k_{B} T \log \left(\mathcal{Z}^{(G S)}\right)
$$

and the total particle number, $N=\partial \log \left(\mathcal{Z}^{(G S)}\right) / \partial(\beta \mu) \equiv \sum_{i} \tilde{n}_{i}^{(G S)}$, where

$$
\tilde{n}_{i}^{(G S)}=\frac{\sum_{n=0}^{\xi} n e^{n \beta\left(\mu-\epsilon_{i}\right)}}{\sum_{n=0}^{\xi} e^{n \beta\left(\mu-\epsilon_{i}\right)}}=\frac{1}{e^{\beta\left(\epsilon_{i}-\mu\right)}-1}-\frac{\xi+1}{e^{(\xi+1) \beta\left(\epsilon_{i}-\mu\right)}-1}
$$

is the average population of the single-particle level $i$. From the Eqs. (12a), we can calculate all the thermodynamics of the system.

At this point the differences between FES and GS become clear. We can split the GS system also into species, like the FES system. Although in both statistics the maximum number of particles per species is the same (provided that the species have the same dimensions), the number of microconfigurations is different in general. This has been calculated in several papers, e.g. [1, 3, 24]. If in the species $i$ of a GS system 
we have, like in FES, $G_{i}$ states and $N_{i}$ particles, and we denote by $R_{i} \equiv\left[G_{i} /(\xi+1)\right]$ the greatest integer contained in $G_{i} /(\xi+1)$, then the number of microconfigurations in which the particles can be distributed in the species $i$ is

$$
W_{G S}\left(G_{i}, N_{i}\right)=\sum_{r=0}^{R}(-1)^{r} \frac{G_{i}\left[G_{i}+N_{i}-(\xi+1) r-1\right] !}{r !\left(G_{i}-r\right) !\left[N_{i}-(\xi+1) r\right] !} .
$$

Expression (13) is different, in general, from Eq. (4a), although it reduces to it in special cases, like e.g. when $\xi \geq G_{i}$ in (13) and $\alpha=0$ in (4a).

These differences are reflected in the thermodynamic properties of GS and FES systems, a fact which is particularly obvious in systems with constant density of states.

A transition rate between different species in GS would be rather difficult to write, since it depends on how many states in the target species are fully occupied. Therefore for GS systems we shall consider just transitions from one state to another, as discussed in the beginning of this section.

The main thermodynamic quantities of GS have been calculated for example in Ref. [2]. In the next section we will recover these results by Monte Carlo simulations.

\section{Numerical implementation}

In order to simulate the time evolution of the system and, in the same time, to validate numerically the transition probabilities introduced in Section 3, we take as model system the $d \mathrm{D}$ ideal gas, contained in a box of linear dimension $L$, with the density of oneparticle states

$$
g(\epsilon)=\frac{\hbar^{2}}{2 m}\left(\frac{L}{2 \pi}\right)^{d} \frac{\pi^{d / 2}}{\Gamma(d / 2)} \epsilon^{d / 2-1} .
$$

We split the energy axis into "short" segments, $\left(\epsilon_{i}, \epsilon_{i+1}\right), i=0,1, \ldots$, which define the particle species. The segments are chosen in such a way that each species contains the

same number of single particle states, $G=\int_{\epsilon_{i}}^{\epsilon_{i+1}} g(\epsilon) d \epsilon$, for any $i$. We shall consider only the case $\alpha_{i j}=\alpha \delta_{i j}$. For the convenience of numerical calculations, we require that $G / \alpha$ is an integer, while for GS we take $G=1$.

We set the total number of particles in each FES system to be $N$ and we fix the energy scale by setting the Fermi energy in each system. For the numerical calculations, we have to fix the highest energy in the system, $\epsilon_{\max }$, so that the contribution coming from the higher energy levels can be neglected in the thermodynamic results.

In this paper we shall consider only $1 \mathrm{D}$ and $2 \mathrm{D}$ systems, so we define a scaling temperature, $T_{0}$, by $\rho \lambda^{d}\left(T_{0}\right)=1$, where $\rho=N / L^{d}$ is the $d \mathrm{D}$ particle density and $\lambda(T)=h / \sqrt{2 \pi m k_{B} T}$ is the thermal length. This gives

$$
T_{0}=\frac{N^{2 / d}}{L^{2}}\left(\frac{h^{2}}{2 \pi m k_{B}}\right)^{1 / 2}, d=1,2 .
$$

Concretely, we shall take $\epsilon_{\mathrm{F}}(\alpha) \equiv \mu(T=0 ; \alpha)=\alpha$ and $\epsilon_{\max }=30$, which would permit us to vary the temperature in a range between zero and $2 T_{0}$, without any observable effect on the thermodynamic quantities. We set the energy unit to $\epsilon_{\mathrm{F}}(\alpha=1)$. 
In FES systems we cannot use directly the transition rates given in Eqs. (8) and (10), because they involve in general too big numbers which cannot be calculated numerically. The quantities that are interesting for us are actually the relative transition rates, $T_{p, j i}\left(N_{j}-1, N_{i}\right) / T_{p, i j}\left(N_{i}, N_{j}-1\right)$. Moreover, in order to obtain a common description for both, bosons and fermions, we define $G_{0 i} \equiv G_{i}+\alpha N_{i}$, for bosons, and $G_{0 i} \equiv G_{i}-(1-\alpha) N_{i}+1$ for fermions. In these notations the number of microconfigurations for the species $i$ becomes [7, 25]

$$
W\left(G_{i}, N_{i}\right)=\frac{\left[G_{0 i}+(1-\alpha) N_{i}-1\right] !}{N_{i} !\left(G_{0 i}-\alpha N_{i}-1\right) !}
$$

for any type of particles. Therefore from now on we shall omit the subscripts $B, F$, or $p$ in our notations.

To calculate the ratio $T_{p, j i}\left(N_{j}-1, N_{i}\right) / T_{p, i j}\left(N_{i}, N_{j}-1\right)$ we take its logarithm and, by noticing from Eqs. (8) and (10) that $T_{p, j i}\left(N_{j}-1, N_{i}\right)=T_{p, i j}\left(N_{i}-1, N_{j}\right)$, we write

$$
\begin{aligned}
\log \left[\frac{T_{j i}\left(N_{j}-1, N_{i}\right)}{T_{i j}\left(N_{i}, N_{j}-1\right)}\right] & =\log \left[T_{i j}\left(N_{i}-1, N_{j}\right)\right]-\log \left[T_{i j}\left(N_{i}, N_{j}-1\right)\right] \\
& \approx-\frac{\partial \log \left[T_{i j}\left(N_{i}, N_{j}\right)\right]}{\partial N_{i}}+\frac{\partial \log \left[T_{i j}\left(N_{i}, N_{j}\right)\right]}{\partial N_{j}} \\
& \approx \log \left\{\frac{\frac{\left[1+(1-\alpha) \tilde{n}_{j}\right]^{1-\alpha}\left(1-\alpha \tilde{n}_{j}\right)^{\alpha}}{\tilde{n}_{j}} e^{-\beta \epsilon_{j}}}{\frac{\left[1+(1-\alpha) \tilde{n}_{i}\right]^{1-\alpha\left(1-\alpha \tilde{n}_{i}\right)^{\alpha}}}{\tilde{n}_{i}} e^{-\beta \epsilon_{i}}}\right\}
\end{aligned}
$$

where $\tilde{n}_{i} \equiv N_{i} / G_{i}$ and in obtaining the last line we used the Stirling approximation, $\log N ! \approx N \log (N / e)$. Therefore, from now on we shall use

$$
T_{j i}\left(N_{j}, N_{i}\right)=\tilde{n}_{i}\left[1+(1-\alpha) \tilde{n}_{j}\right]^{1-\alpha}\left(1-\alpha \tilde{n}_{j}\right)^{\alpha},
$$

up to the relative canonical factor, $e^{\beta\left(\epsilon_{i}-\epsilon_{j}\right)}$. One can understand the derived transition rates as follows: a new state is proposed by moving one particle from species $i$ to species $j$ with a step probability which depends on the instantaneous average occupations of the two species, i.e. $\sim \tilde{n}_{i}\left[1+(1-\alpha) \tilde{n}_{j}\right]^{1-\alpha}\left(1-\alpha \tilde{n}_{j}\right)^{\alpha}$, and it is accepted with the usual Metropolis probabilities, $\min \left(1, \exp \left(\beta\left(\epsilon_{i}-\epsilon_{j}\right)\right)\right.$. In this way the dynamics of FES systems can be viewed as the dynamics of classical ideal gas, but with new step probabilities which stem from a generalized exclusion principle. This picture is consistent with the one described in Ref. [5] for Bose and Fermi systems. From Eq. (17) or (18) it becomes obvious that when equilibrium is reached, all the transition rates become equal and therefore the DBE is satisfied, as shown in Section 3.1 .

In the numerical calculations we shall take $G_{0 i} \equiv G=120$ for any $i$ and $N=12000$. These values are large enough to obtain the analytical equilibrium distribution with high accuracy. Nevertheless, a discussion about the species' size is also given below. Using these parameters, the considered systems are proved to be very well described analytically by continuous models, so that a direct comparison is possible.

Typical results for equilibration in two-dimensional systems with the FES parameter $\alpha$ taking the values $0,1 / 4,1 / 2,3 / 4,1$ are indicated in Fig. 1. The systems are first prepared in their ground state $(T=0 K)$ configurations and the dynamics 


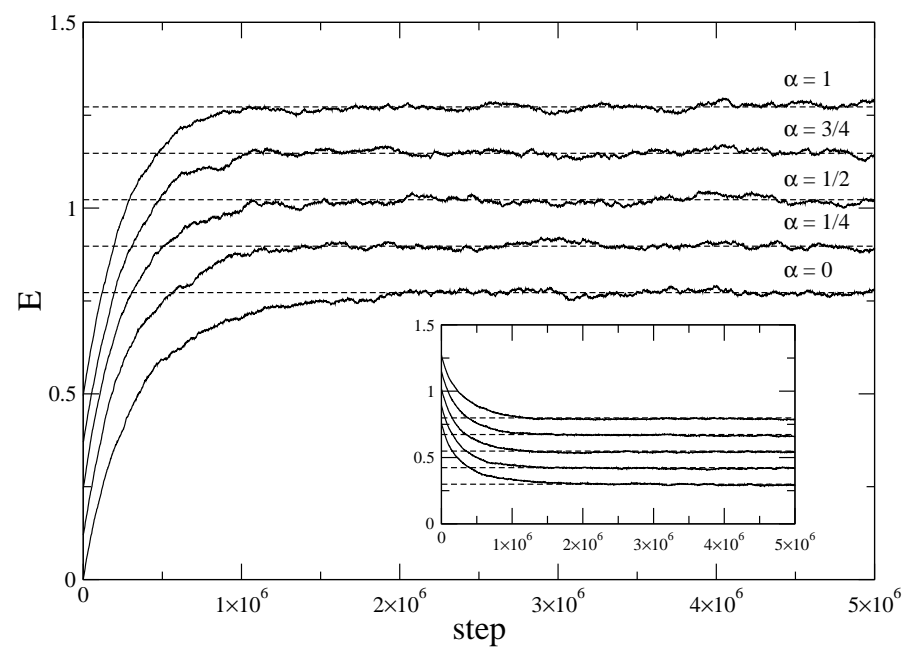

Figure 1. Typical equilibration of two-dimensional FES systems, by raising (main plot) or lowering (inset) the temperature. The horizontal lines mark the average equilibrium energy of each system, calculated analytically.

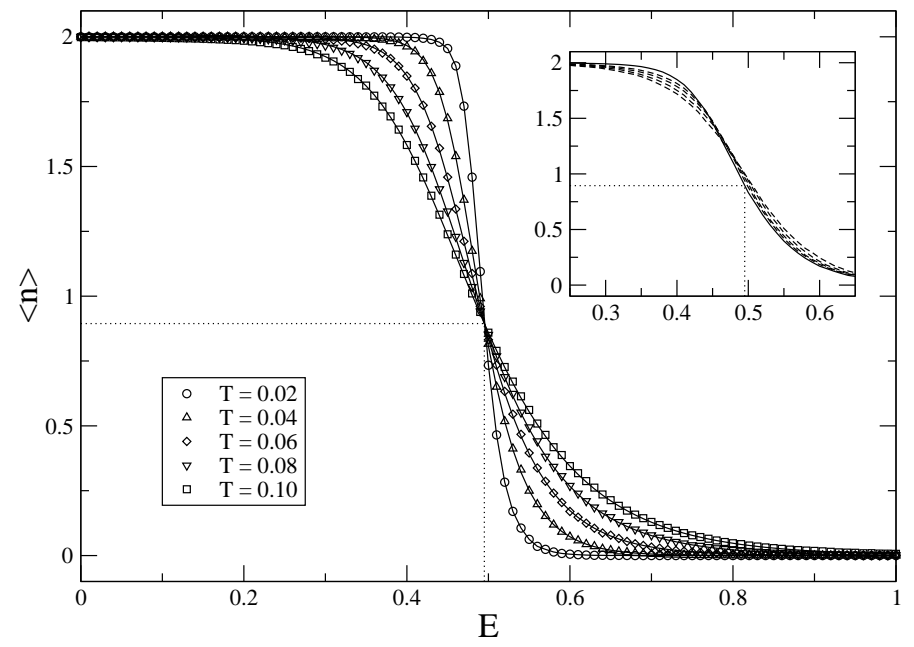

Figure 2. Main plot: Analytical (solid lines) and numerical (symbols) data for a twodimensional system with a number of single particle states in a species $G=120$, for the five indicated temperatures. Inset: Numerical data for $T=0.06 T_{0}$, with $G=120$ (solid) and $G=1,2,4,8$ (dashed), where the largest deviation corresponds to $G=1$. Here the symbols have been dropped for convenience.

towards equilibrium is depicted at $T=T_{0}$. The inset contains the opposite situation i.e. the systems are quenched from $T_{0}$ to $0.5 T_{0}$. In this case, the initial configuration is a random configuration drawn according to the equilibrium distribution, calculated analytically at $T_{0}$. In both cases, i.e. either increasing or decreasing the temperature, after a number of $\sim 10^{6}$ steps, the energy of systems gets close to and starts oscillating around the equilibrium value, as calculated analytically. 


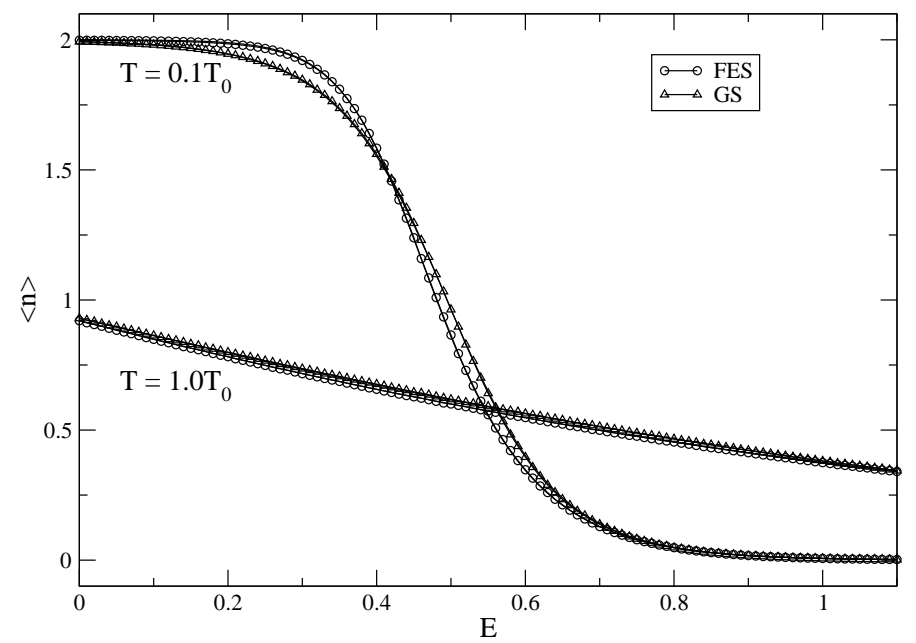

Figure 3. Comparison between FES and GS for semions $(\alpha=1 / \xi=1 / 2)$, for low $\left(T=0.1 T_{0}\right)$ and high temperatures $\left(T=T_{0}\right)$. Analytical data is represented by solid lines and numerical data by symbols.
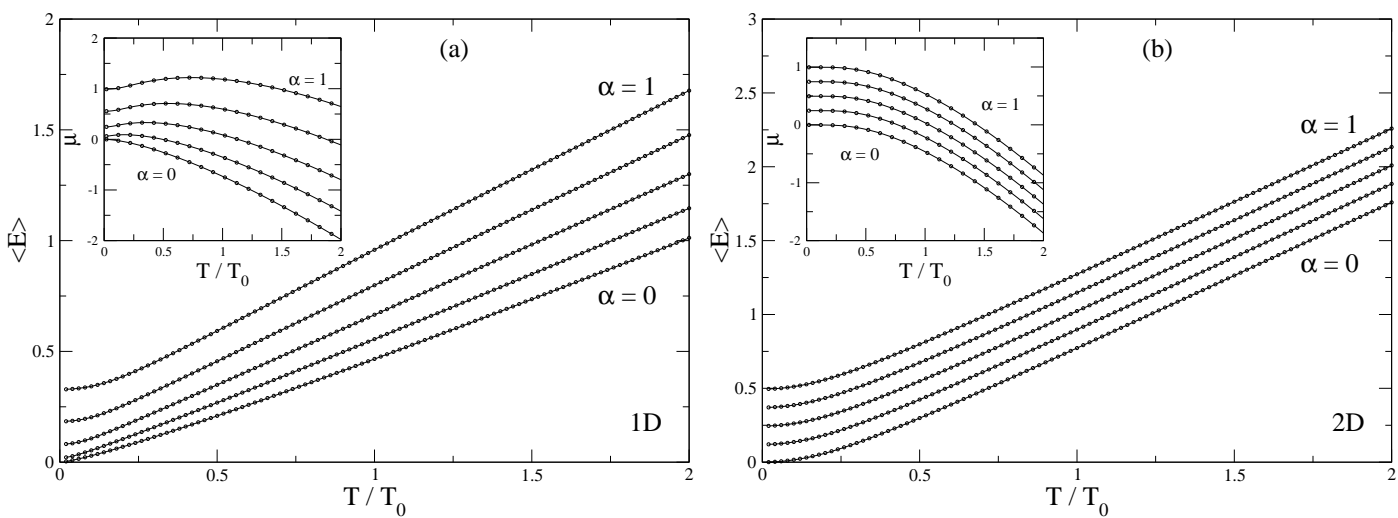

Figure 4. Average total energy vs. temperature (symbols) for $d=1$ (a) and $d=2$ (b) for FES systems with $\alpha=0,1 / 4,1 / 2,3 / 4,1$. Inset: Temperature dependence of the chemical potential. Solid lines represent analytical calculations using the corresponding continuous model.

Figure 2 shows the average occupation of a two-dimensional system with $\alpha=1 / 2$, which is calculated performing time averages after the system has reached equilibrium. The system is first very well thermalized for number of $N_{0}=10^{9}$ steps and then the time average is obtained using an equal number of additional steps. We included every $1000^{\text {th }}$ microconfiguration in the time-average, i.e. we considered a total of $10^{6}$ microconfigurations.

The data is fitted very well by analytical calculations using the corresponding continuous model. From Eq. (17) of Ref. [7], one can see that in the degenerate limit, i.e. low temperatures, the different distributions cross at the point $\left(E=\mu_{T=0}=0.495,\langle n\rangle=\right.$ $\sqrt{4 / 5})$, as it is indicated by dotted lines, the small deviation of 0.005 in $\mu_{\mathrm{T}=0}$, being caused by the finite spacing between the energy levels. In FES systems, if one decreases 


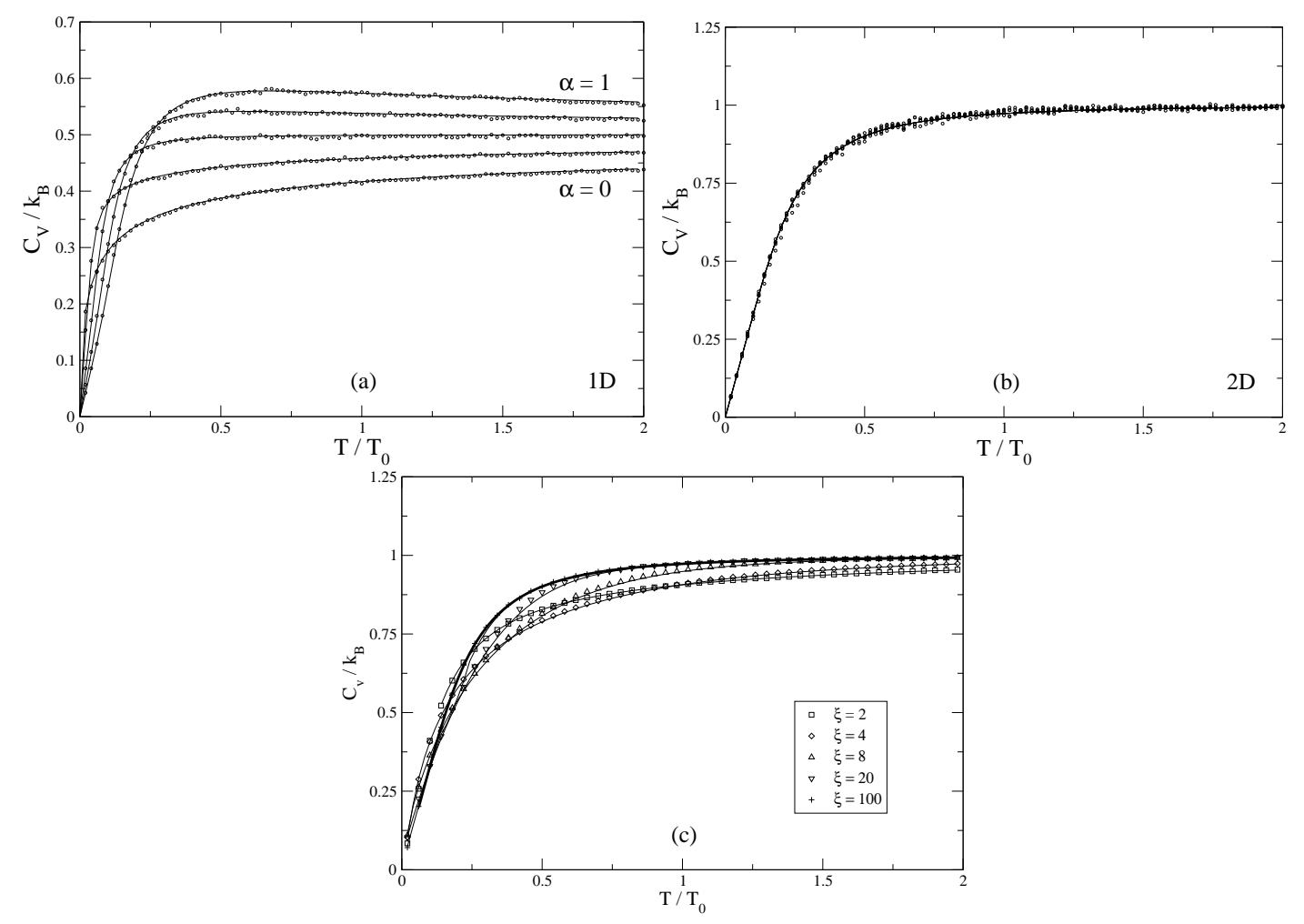

Figure 5. Heat capacity vs. temperature (symbols) for one-dimensional (a) and twodimensional (b) FES systems, and for two-dimensional GS systems (c). Solid lines represent analytical calculations using the corresponding continuous model.

the number of single particle states in a species, $G$, the average distributions deviate, as it is shown in the inset. For the limiting case, $G=1$, it is established that the average FES distributions for different temperatures in the degenerate limit cross at $\langle n\rangle=1$. Therefore we emphasize the high importance of the species' size in obtaining the correct FES distribution.

In Fig. 3 we analyze comparatively the two statistics, FES and GS. For this we plot the equilibrium distributions for semions $(\alpha=1 / \xi=1 / 2)$. Although in the high temperature limit the distributions become both identical with the classical Maxwell Boltzmann distribution, there are visible differences at low temperatures. One particular difference is that GS equilibrium distributions in the low temperature limit cross at $\langle n\rangle=\xi / 2$, which is not the case for the FES systems.

Next we perform long runs to obtain thermodynamic quantities with good accuracy. As before, we take $N_{0}=10^{9}$ steps to reach equilibrium and another $N_{1}=10^{10}$ steps to obtain the time averages. The results for the average energy and chemical potential as function of temperature are depicted in Figs. 4. The chemical potential is extracted as a fit parameter for the average distribution obtained numerically, in order to match the analytical result for the mean occupation. The characteristics related to the dimensionality of the systems are clearly visible: for $d=2$ case the data is only vertically shifted, which is a consequence of the thermodynamic equivalence of systems 
of constant density of states and any statistics [11, 12, 13, 15].

The heat capacity is calculated from the fluctuations of the total energy, $C_{\mathrm{v}}=$ $\left(\left\langle E^{2}\right\rangle-\langle E\rangle^{2}\right) /\left(k_{\mathrm{B}} T^{2}\right)$. The data plotted in Fig. 5 confirms again the correctness of our approach for the proposed step probabilities for FES and GS systems. As expected, for FES systems and $d=2$, the data for all values $\alpha$ collapses on the same curve. By contrast, the thermodynamic behavior of GS systems is no longer equivalent with respect to the $\xi$ parameter. The thermodynamic equivalence of the $2 \mathrm{D}$ systems suggests that FES is a more natural extension of Fermi and Bose statistics.

\section{Conclusions}

The present approach introduces a stochastic model for the non-equilibrium dynamics of systems which obey generalized statistics, such as fractional exclusion statistics (FES) and Gentile statistics (GS). The model has at its core a time-dependent Markov chain in the microconfiguration state space, which generalizes previous results from Ref. [5]. In the derivation of the stochastic transition probabilities, a division can be made which separates them into acceptance probabilities, corresponding to transition probabilities in a classical ideal gas (i.e. Metropolis probabilities), and step probabilities, which account for the generalized exclusion principle employed.

The obtained probabilities are tested extensively by Monte Carlo simulations on one- and two-dimensional FES and GS systems. The numerical results reflecting several thermodynamic quantities overlap very well with the reference data, calculated analytically. We point out here the crucial role played by the size of individual species in the FES systems, as well as the fundamental differences which appear in the thermodynamics properties of the two-dimensional FES and GS systems.

The Monte Carlo approach to FES could yield new insights in the time evolution of finite quantum many-body systems regarded as FES systems, indicating potential differences related to out-of-the-equilibrium phenomena.

\section{References}

[1] G. Gentile. Nuovo Cim., 17:493, 1940.

[2] S. Katsura, K. Kaminishi, and S. Inawashiro. J. Math. Phys., 11:2691, 1970.

[3] R. L. Ponczek and C. C. Yan. Revista Brasileira de Fisica, 6:471, 1976.

[4] F. D. M. Haldane. Phys. Rev. Lett., 67:937, 1991.

[5] I. Guastella, L. Bellomonte, and R. M. Sperandeo-Mineo. J. Stat. Mech., 2009:P02021, 2009.

[6] D. V. Anghel. EPL, 87:60009, 2009. arXiv:0906.4836,

[7] Yong-Shi Wu. Phys. Rev. Lett., 73:922, 1994.

[8] S. B. Isakov. Phys. Rev. Lett., 73(16):2150, 1994.

[9] D. V. Anghel. J. Phys. A: Math. Theor., 40:F1013, 2007. arXiv:0710.0724,

[10] D. V. Anghel. EPL, 90:10006, 2010. arXiv:0909.0030.

[11] F. C. Auluck and D. S. Kothari. Proc. Cambridge Philos. Soc., 42:272, 1946.

[12] Robert M. May. Phys. Rev., 135:A1515, 1964.

[13] M. Howard Lee. Phys. Rev. E, 55:1518, 1997.

[14] M. H. Lee and J. Kim. Physica A, 304:421, 2002. 
[15] D. V. Anghel. J. Phys. A: Math. Gen., 35:7255, 2002.

[16] D. V. Anghel. J. Phys. A: Math. Gen., 39:4787, 2006.

[17] D. V. Anghel. Phys. Lett. A, 372:5745, 2008. arXiv:0710.0728.

[18] D. V. Anghel. Rom. J. Phys., 54:281, 2009. arXiv:0804.1474,

[19] D. Bernard and Y. S. Wu. In M. L. Ge and Y. S. Wu, editors, New Developments on Integrable Systems and Long-Ranged Interaction Models, page 10. World Scientific, Singapore, 1995. cond-mat/9404025.

[20] B. Sutherland. Phys. Rev. B, 56:4422, 1997.

[21] M. V. N. Murthy and R. Shankar. Phys. Rev. Lett., 73:3331, 1994.

[22] D. Sen and R. K. Bhaduri. Phys. Rev. Lett., 74:3912, 1995.

[23] D. V. Anghel. Rom. Rep. Phys., 59:235, 2007. cond-mat/0703729.

[24] M. E. Fisher. Am. J. Phys., 29:49, 1961.

[25] M. V. N. Murthy and R. Shankar. Phys. Rev. B, 60:6517, 1999. 